University of Wollongong

Research Online

Faculty of Social Sciences - Papers (Archive) Faculty of Arts, Social Sciences \& Humanities

2008

Effects of fully-established Sure Start Local Programmes on 3-year-old children and their families living in England: a quasi-experimental observational study

Edward Melhuish

University of Wollongong, melhuish@uow.edu.au

Jay Belsky

University of London

Alastair H. Leyland

University of Glasgow

Jacqueline Barnes

University of London

Follow this and additional works at: https://ro.uow.edu.au/sspapers

Part of the Education Commons, and the Social and Behavioral Sciences Commons

Research Online is the open access institutional repository for the University of Wollongong. For further information contact the UOW Library: research-pubs@uow.edu.au 


\title{
Effects of fully-established Sure Start Local Programmes on 3-year-old children and their families living in England: a quasi-experimental observational study
}

\author{
Abstract \\ Background
}

Sure Start Local Programmes (SSLPs) are area-based interventions to improve services for young children and their families in deprived communities, promote health and development, and reduce inequalities. We therefore investigated whether SSLPs affect the wellbeing of 3-year-old children and their families.

\section{Methods}

In a quasi-experimental observational study, we compared 5883 3-year-old children and their families from 93 disadvantaged SSLP areas with 1879 3-year-old children and their families from 72 similarly deprived areas in England who took part in the Millennium Cohort Study. We studied 14 outcomes-children's immunisations, accidents, language development, positive and negative social behaviours, and independence; parenting risk; home-learning environment; father's involvement; maternal smoking, body-mass index, and life satisfaction; family's service use; and mother's rating of area.

\section{Findings}

After we controlled for background factors, we noted beneficial effects associated with the programmes for five of 14 outcomes. Children in the SSLP areas showed better social development than those in the non-SSLP areas, with more positive social behaviour (mean difference $0.45,95 \% \mathrm{Cl} 0.09$ to $0.80, \mathrm{p}=0.01$ ) and greater independence $(0.32,0.18$ to $0.47, p<0.0001)$. Families in SSLP areas showed less negative parenting $(-0.90,-1.11$ to $-0.69, p<0.0001)$ and provided a better home-learning environment $(1.30,0.75$ to $1 \cdot 86, p<0 \cdot 0001)$. These families used more services for supporting child and family development than those not living in SSLP areas $(0 \cdot 98,0.86$ to $1 \cdot 09, p<0 \cdot 0001)$. Effects of SSLPs seemed to apply to all subpopulations and SSLP areas.

\section{Interpretation}

Children and their families benefited from living in SSLP areas. The contrast between these and previous findings on the effect of SSLPs might indicate increased exposure to programmes that have become more effective. Early interventions can improve the life chances of young children living in deprived areas.

\section{Funding}

The Department for Children, Schools and Families.

\section{Keywords}

sure, observational, established, fully, effects, experimental, quasi, england, living, families, their, children, old, year, 3, programmes, local, start, study

\section{Disciplines}

Education | Social and Behavioral Sciences

\section{Publication Details}

Melhuish, E., Belsky, J., Leyland, A. H. \& Barnes, J. (2008). Effects of fully-established Sure Start Local Programmes on 3-year-old children and their families living in England: a quasi-experimental observational study. The Lancet, 372 (9650), 1641-1647. 


\section{Effects of fully-established Sure Start Local Programmes on 3-year-old children and their families living in England: a quasi-experimental observational study}

Edward Melhuish, Jay Belsky, Alastair H Leyland, Jacqueline Barnes, and the National Evaluation of Sure Start Research Team*

\section{Summary}

Background Sure Start Local Programmes (SSLPs) are area-based interventions to improve services for young children and their families in deprived communities, promote health and development, and reduce inequalities. We therefore investigated whether SSLPs affect the wellbeing of 3-year-old children and their families.

Methods In a quasi-experimental observational study, we compared 5883 3-year-old children and their families from 93 disadvantaged SSLP areas with 1879 3-year-old children and their families from 72 similarly deprived areas in England who took part in the Millennium Cohort Study. We studied 14 outcomes-children's immunisations, accidents, language development, positive and negative social behaviours, and independence; parenting risk; home-learning environment; father's involvement; maternal smoking, body-mass index, and life satisfaction; family's service use; and mother's rating of area.

Findings After we controlled for background factors, we noted beneficial effects associated with the programmes for five of 14 outcomes. Children in the SSLP areas showed better social development than those in the non-SSLP areas, with more positive social behaviour (mean difference $0.45,95 \%$ CI 0.09 to $0.80, \mathrm{p}=0.01$ ) and greater independence $(0.32,0.18$ to $0.47, \mathrm{p}<0.0001)$. Families in SSLP areas showed less negative parenting $(-0.90,-1 \cdot 11$ to -0.69 , $\mathrm{p}<0.0001)$ and provided a better home-learning environment $(1 \cdot 30,0 \cdot 75$ to $1 \cdot 86, \mathrm{p}<0 \cdot 0001)$. These families used more services for supporting child and family development than those not living in SSLP areas $(0.98,0.86$ to $1.09, \mathrm{p}<0.0001)$. Effects of SSLPs seemed to apply to all subpopulations and SSLP areas.

Interpretation Children and their families benefited from living in SSLP areas. The contrast between these and previous findings on the effect of SSLPs might indicate increased exposure to programmes that have become more effective. Early interventions can improve the life chances of young children living in deprived areas.

Funding The Department for Children, Schools and Families.

\section{Introduction}

The main aim of Sure Start Local Programmes (SSLPs) is to improve the health and wellbeing of young children living in disadvantaged neighbourhoods by preventing the transmission of inequalities in health, poverty, school failure, and social exclusion between generations. SSLPs were set up between 1999 and 2003 to develop different ways of providing services in deprived communities. The original programmes were areabased, with all young children and their families living in a prescribed geographic area being the targets of the intervention. SSLPs were targeted to $20 \%$ of the most deprived areas in England and programmes were managed by a partnership of health, education, social services, and voluntary sectors. By contrast with more targeted interventions, the SSLPs initially did not have a prescribed set of services, especially not those delineated in a manualised form to promote fidelity of treatment to a prescribed model. Instead, each local programme was responsible for working with the community to improve existing services according to local needs while covering core services-ie, outreach and home visits; support to families and parents; support for good-quality play, learning, and childcare; primary and community health care, and support for children and parents with special needs, but without specification of how services were to be changed. ${ }^{1}$ This approach contrasts greatly with previous interventions shown to be effective, whether childcare-based (eg, the Abecedarian project ${ }^{2}$ ), homebased (the Nurse Family Partnership ${ }^{3}$ or the Positive Parenting programme $\mathrm{e}^{4}$, or a combination of centrebased and home-based (Early Head Start ${ }^{5}$ ).

In the first part of our evaluation, comparing children and families living in 150 SSLP areas and in 50 communities destined to become SSLP areas, we noted small positive and negative effects of SSLPs on 3 -year-old children. ${ }^{6,7}$ Although the less disadvantaged of the predominantly disadvantaged families living in SSLP areas benefited somewhat, adverse effects emerged for the most disadvantaged. Specifically, non-teenage mothers (86\%) living in SSLP areas showed less negative parenting than those in the comparison communities, resulting in their 3-year-old children having fewer behaviour problems and greater social competence. However, children in SSLP areas living in workless households (39\%), in lone-parent families (36\%), or born
Lancet 2008; 372: 1641-47 See Comment page 1610 *Members listed at end of paper Institute for the Study of Children, Families and Social Issues, Birkbeck University of London, London, UK (Prof E Melhuish PhD, ProfJ Belsky PhD, Prof J Barnes PhD); and MRC Social and Public Health Sciences Unit, Glasgow (Prof A H Leyland PhD) Correspondence to: Prof Edward Melhuish, Institute for the Study of Children, Families and Social Issues, Birkbeck University of London, 7 Bedford Square, London WC1 B 3RA, UK e.melhuish@bbk.ac.uk 
For more details about the census see https://www.census. ac.uk/guides/Area_stats.aspx to teenage mothers $(14 \%)$ had lower scores than those in comparison communities for verbal ability, and children born to teenage mothers also had more behaviour problems and less social competence.

SSLPs have evolved, changing their model of service delivery by becoming Sure Start Children's Centres during 2004-06. The changes included clearer specification of services, with a strong emphasis on child

Panel: Description of outcome variables

\section{Physical health}

- Child immunisations: children divided into two categories, those who had had all recommended immunisations and those who had not

- Child accidents: children divided into two categories, those who had had at least one accident requiring medical attention since the child was 9 months old, and those who had not

\section{Child language development}

- British Abilities Scales ${ }^{11}$ naming vocabulary: picture-naming vocabulary subscale

\section{Child social and emotional development}

- Child positive social behaviour: child is generally obedient, can stop and think before acting, sees tasks through to the end, has a good attention span, thinks about other people's feelings, shares readily with other children, is helpful if someone is hurt, upset, or feeling ill, is kind to younger children, often offers to help others ${ }^{12}$

- Child negative social behaviour: child often complains of headaches, stomach aches, or sickness, is often unhappy or tearful, has many fears and is easily scared, often fights with other children or bullies them, often argues with adults ${ }^{12}$

- Independence: child likes to work things out by himself or herself, does not need much help with tasks, chooses activities independently, persists with difficult tasks, and can move to a new activity after finishing a task ${ }^{13}$

\section{Parenting and family functioning}

- Parenting risk index: made up of six parenting variables-ie, observer's rating of mother's responsiveness to child (praising, responding, showing affection), observer's rating of mother's acceptance of child (not observing, scolding or derogating, spanking, physically restraining) ${ }_{1}^{14}$ parent-child conflict (parent-child struggles, child easily angry with parent, conflict with discipline), parent-child closeness (affectionate relationship, child seeks comfort, shares feelings), ${ }_{1}^{15}$ harsh discipline (ignoring, smacking, shouting, punishing) ${ }_{1}^{16}$ home chaos (disorganised, noisy) ${ }^{17}$

- Home learning environment: made up of six items of frequency of learning opportunities for child in home-ie, taken to library, helped to learn or play with alphabet, helped to learn or play with numbers, child read to, taught songs and rhymes, child paints and draws ${ }^{18}$

- Father's involvement: how often the father plays with the child and how often he gets the child ready for bed in the evening

\section{Maternal wellbeing}

- Currently smoking: mothers who smoke tobacco versus those who do not

- Life satisfaction: how satisfied or dissatisfied respondent is about the way life has turned out

- Mother's body-mass index

Service use

- Family's service use: number of different types of services used in past 12 months

Local-area measurements

- Mother's area rating: respondent's rating of local area

wellbeing and the need to reach the most vulnerable, and adjustment of service provision to the degree of family disadvantage. ${ }^{1}$ In view of these changes and that 3-yearold children studied in the first part of the evaluation were not exposed to SSLPs during their entire lives, a second part of the evaluation was undertaken, with follow-up at age 3 years for many of the 9-month-old infants included in the first part of the study. These children and their families were compared with those enrolled in the Millennium Cohort Study ${ }^{8}$ in which the children had also been followed up since 9 months of age. Selection of children and families for the Millennium Cohort Study ${ }^{8}$ from the larger cohort sample was based on areas similar to those of the SSLP sample (ie, deprived) but not benefiting from an SSLP.

Our aim in this study was to compare the wellbeing of 3-year-old children and their families living in SSLP areas and in similar non-SSLP areas.

\section{Methods}

\section{Study design and participants}

We randomly selected participants living in 150 SSLP areas in England from the Child Benefit Register; 12575 infants aged 9 months and their families were enrolled in a study of children, families, and communities, representing a response rate of $84.4 \%$. Of the children seen at age 9 months, 11118 and their families were randomly selected for home visits when the child was 3 years of age (funding limitation precluded follow-up of all 12575 children); 9192 of these $(82.7 \%)$ took part in this assessment. Of those who did not take part, 388 refused $(3 \cdot 5 \%), 1484(13 \cdot 3 \%)$ could not be contacted because they had moved and were untraceable, and $54(<1 \%)$ were not seen for various other reasons.

As controls, children and their families living in non-SSLP areas were chosen from the Millennium Cohort Study. ${ }^{8}$ To compare families from non-SSLP areas who were as similar as possible to those in the SSLP areas studied, we selected areas in England in which children from the Millennium Cohort Study lived but which did not have SSLPs by use of propensity-score matching 9 for having similar area characteristics to SSLPs on the basis of 85 variables from the Indices of Multiple Deprivation ${ }^{10}$ and the 2001 census. The reasons why non-SSLP areas had not received SSLPs despite being appropriately deprived are unknown. For primary comparisons, the SSLP sample was restricted to those children and families residing in areas most like the non-SSLP areas; we had to exclude the most disadvantaged SSLP areas because there were no similarly deprived areas in the Millennium Cohort Study sample. Thus, the final samples used for primary comparisons consisted of 5883 children and their families in 93 (of the original 150) SSLP areas and 1879 children and their families in 72 non-SSLP areas. In secondary analyses, children and families from the SSLP areas excluded from the 
primary analyses were compared with those in the 93 SSLP areas in the primary analyses. No information was gathered on non-respondents.

Parents or guardians provided written informed consent. Multiregion ethics approval was obtained from the South West Multicentre Research Ethics Committee.

\section{Procedures and measurements}

During the 90 min home visits, all recruited families provided detailed information about child and family functioning when children were aged 9 months and 3 years. The research teams coordinated many methods and measurements so that SSLP and non-SSLP areas could be compared, though data were gathered by separate research teams, with those for non-SSLP areas being gathered, on average, 2 years before those for the SSLP areas. We did analyses specifically to test whether such a timing difference might affect results. We derived data from parents' reports and a standard assessment of English-speaking children's language development. Family demographic and background information served mainly as control variables in the analyses. Additionally, area-level data from the Indices of Multiple Deprivation ${ }^{10}$ and the 2001 census were factor-analysed to create seven area-level factors that provided additional statistical control in comparisons.

To ensure that no selection bias entered into the choice of analyses, we analysed all outcomes that could be compared because they were measured identically across samples, irrespective of their importance, to test for SSLP versus non-SSLP differences. These 14 outcomes were children's immunisations, accidents, language development, positive and negative social behaviour, and independence; parenting risk; home learning environment; father's involvement; maternal smoking, body-mass index (BMI), and life satisfaction; family's service use; and mother's rating of area. We derived seven of 14 outcomes, including all child development and parenting measures (except father involvement) and mother's BMI, from standard measures used in other studies, ${ }^{11-18}$ and the other seven outcomes from the parents' report (panel). Table 1 shows the characteristics of people in SSLP and non-SSLP areas. Some demographic differences between the samples indicate greater disadvantage in the SSLP sample (ie, more mothers with cognitive difficulties, more lone parents, more workless households), whereas other differences in ethnicity indicate potentially less disadvantage in the SSLP sample (ie, more white families, fewer homes with English as second language). In both samples, some children and families were seen at 9 months but were not seen again at age 3 years, and $3 \cdot 6 \%$ of data were missing overall. Missing data were most prevalent for the outcomes of British Abilities Scale naming vocabulary $(19 \%)$, parenting risk $(21 \%)$, and maternal BMI (15\%); all other variables had less than $5 \%$ missing data. The risk of biased comparisons was increased by

\begin{tabular}{|lccc|}
\hline & SSLP area (n=5883) & $\begin{array}{c}\text { Non-SSLP area } \\
(\mathbf{n}=1879)\end{array}$ & p value \\
\hline Child's sex (male) & $2955(50 \%)$ & $948(50 \%)$ &.. \\
Child's ethnic origin &.. &.. & $<0.0001$ \\
White & $4962(84 \%)$ & $1326(71 \%)$ &.. \\
Mixed & $246(4 \%)$ & $97(5 \%)$ &.. \\
Indian & $64(1 \%)$ & $168(9 \%)$ &.. \\
Pakistani & $203(3 \%)$ & $96(5 \%)$ &.. \\
Bangladeshi & $49(<1 \%)$ & $41(2 \%)$ &.. \\
Black Caribbean & $78(1 \%)$ & $42(2 \%)$ &.. \\
Black other & $164(3 \%)$ & $52(3 \%)$ &.. \\
Other & $117(2 \%)$ & $57(3 \%)$ &.. \\
Home language English & $5218(89 \%)$ & $1456(77 \%)$ & $<0.0001$ \\
Mother teenage $(<20$ years) at birth & $614(10 \%)$ & $183(10 \%)$ &.. \\
Maternal cognitive difficulties & $539(9 \%)$ & $135(7 \%)$ & $<0.002$ \\
Below poverty line & $2736(47 \%)$ & $921(49 \%)$ &.. \\
Highest occupation in household &.. &.. & $<0.04$ \\
Management/professional & $1328(23 \%)$ & $429(23 \%)$ &.. \\
Intermediate & $524(9 \%)$ & $153(8 \%)$ &.. \\
Small employer & $395(7 \%)$ & $150(8 \%)$ &.. \\
Lower supervisory/technical & $524(9 \%)$ & $208(11 \%)$ &.. \\
Semi-routine & $720(12 \%)$ & $248(13 \%)$ &.. \\
Routine & $524(9 \%)$ & $176(9 \%)$ &.. \\
Unemployed & $1868(32 \%)$ & $515(27 \%)$ &.. \\
Lone parent & $1521(26 \%)$ & $408(22 \%)$ & $<0.0001$ \\
Workless household & $1664(28 \%)$ & $429(22 \%)$ & $<0.0001$ \\
Highest education in household &.. &.. & $<0.003$ \\
Degree/higher education & $1659(28 \%)$ & $477(25 \%)$ &.. \\
A level & $1765(30 \%)$ & $628(33 \%)$ &.. \\
O level/GCSE & $1351(23 \%)$ & $467(25 \%)$ &.. \\
Other & $468(8 \%)$ & $86(5 \%)$ &.. \\
None & $640(11 \%)$ & $221(12 \%)$ &.. \\
& & &. \\
& & & \\
\hline
\end{tabular}

Data are number (\%), unless otherwise indicated. SSLP=Sure Start Local Programme.

Table 1: Summary of demographic characteristics

missing data. However, characteristics of families lost to follow-up and with missing data were similar in both samples, which suggests that any differences between SSLP and non-SSLP areas were unlikely to be a function of attrition or missing data.

\section{Statistical analysis}

We used multilevel models ${ }^{19}$ because children and families were clustered within areas. Since SSLPs served entire communities, we did the analyses on the assumption of intention to treat. We adjusted all analyses for the background characteristics of the child, family, and area (table 1), and for time of measurement (ie, months since January, 2000), before evaluating differences between SSLP and non-SSLP areas. To assess whether different subpopulations were affected differently by SSLPs, as noted in our previous investigation, ${ }^{7}$ we tested selected two-way interactions of SSLP with child sex, ethnic group, teenage 


\begin{tabular}{|c|c|c|c|c|c|c|c|}
\hline & \multicolumn{6}{|c|}{ Estimated effect of SSLP versus non-SSLP } & \multirow{3}{*}{$\begin{array}{l}\text { Effect size } \\
\text { (SD units) }\end{array}$} \\
\hline & \multicolumn{3}{|l|}{ Complete data } & \multicolumn{3}{|l|}{ Imputed data } & \\
\hline & Odds ratio $(95 \% \mathrm{Cl})$ & Mean difference $(95 \% \mathrm{Cl})$ & p value & Odds ratio $(95 \% \mathrm{Cl})$ & Mean difference $(95 \% \mathrm{Cl})$ & p value & \\
\hline \multicolumn{8}{|l|}{ Physical health } \\
\hline Child had all immunisations & $0.61(0.27$ to 1.35$)$ & .. & 0.22 & $0.63(0.30$ to 1.33$)$ & .. & 0.22 & .. \\
\hline Child had accident(s) & $1.55(1.07$ to 2.24$)$ & .. & 0.02 & $1.29(0.89$ to 1.86$)$ & .. & $0 \cdot 18$ & .. \\
\hline \multicolumn{8}{|l|}{ Child development } \\
\hline BAS naming vocabulary & .. & $-0.49(-1.52$ to 0.54$)$ & $0 \cdot 35$ & .. & $-0.43(-1.31$ to 0.44$)$ & 0.33 & .. \\
\hline Child positive social behaviour & .. & $0.45(0.09$ to 0.80$)$ & 0.01 & .. & $0.52(0.18$ to 0.86$)$ & $<0.0001$ & 0.19 \\
\hline Child negative social behaviour & .. & $-0.02(-0.20$ to 0.16$)$ & 0.86 & .. & $-0.04(-0.16$ to 0.08$)$ & 0.49 &.. \\
\hline Independence & .. & 0.32 (0.18 to 0.47$)$ & $<0.0001$ & .. & $0.30(0.06$ to 0.53$)$ & $<0.0001$ & 0.17 \\
\hline \multicolumn{8}{|l|}{ Parenting } \\
\hline Parenting risk index & .. & $-0.90(-1.11$ to -0.69$)$ & $<0.0001$ & .. & $-0.81(-1.27$ to -0.90$)$ & $<0.0001$ & $0 \cdot 44$ \\
\hline Home-learning environment & .. & $1.30(0.75$ to 1.86$)$ & $<0.0001$ & .. & $1.96(0.81$ to 1.96$)$ & $<0.0001$ & 0.27 \\
\hline Father involvement & .. & $-0.09(-0.24$ to 0.05$)$ & 0.21 & .. & $-0.06(-0.21$ to 0.08$)$ & 0.40 & .. \\
\hline \multicolumn{8}{|l|}{ Maternal wellbeing } \\
\hline Currently smoking & $0.97(0.81$ to 1.17$)$ & .. & 0.75 & $0.96(0.81$ to 1.14$)$ & .. & 0.64 & .. \\
\hline Life satisfaction &.. & $-0.02(-0.20$ to 0.16$)$ & 0.81 &.. & $0.07(-0.10$ to 0.23$)$ & 0.44 & .. \\
\hline Body-mass index & .. & $0.05(-0.36$ to 0.47$)$ & 0.80 & .. & $0.07(-0.27$ to 0.42$)$ & 0.68 & .. \\
\hline Family's service use & .. & 0.98 (0.86 to 1.09$)$ & $<0.0001$ & .. & 0.90 (0.67 to 1.12$)$ & $<0.0001$ & 0.53 \\
\hline Mother's rating of area & .. & $0.05(-0.05$ to 0.14$)$ & $0 \cdot 35$ & .. & $0.08(-0.01$ to 0.17$)$ & 0.09 & .. \\
\hline
\end{tabular}

motherhood, lone parenthood, workless or working household, and income deprivation. Whenever a significant interaction was found, we used follow-up tests to compare the subgroups in SSLP and non-SSLP samples. Additionally, since some SSLP areas were excluded from SSLP and non-SSLP comparisons, the equivalence of children and families in SSLP areas included and excluded from the primary analyses was tested (after adjustment for demographic variation). If included and excluded SSLP children and their families proved equivalent, the results should be generalisable across SSLPs. To minimise possible bias from missing data, we used multiple imputation for missing data ${ }^{20}$ (IVEware $^{21}$ ); we used all available data to create ten imputed datasets and then analysed and combined the results. Use of complete-cases data avoids assumptions about missing data but might introduce bias if families with missing data differ from those with full data. The imputed data include all families but questions about the validity of imputation arise. To accommodate these issues, we did all analyses with both complete-cases data and multiply-imputed data, and only those that were significant with both complete and imputed data were judged dependable. The statistical software used was SAS (version 9.1). ${ }^{22}$ Table 2 shows the results from both complete and imputed data analyses. Effect sizes were calculated as the mean difference in SD units between SSLP and non-SSLP samples adjusted for covariates.

\section{Role of the funding source}

The sponsor directed the research design, precluding a randomised control trial and necessitating a quasiexperimental design. The sponsor's Sure Start unit and the unit's scientific advisory board reviewed all major research decisions (ie, instrumentation, sampling, analysis). The sponsor reviewed this report before submission for accuracy of findings only. The authors had full access to all data and had final responsibility for the decision to submit for publication.

\section{Results}

We noted significant differences, irrespective of covariate effects, between SSLP and non-SSLP areas in five of 14 outcomes analysed, all five indicating positive SSLP effects (table 2). In SSLP areas, children showed better social development, with more positive social behaviour and greater independence, and parents showed less risk of negative parenting and provided a better home-learning environment (table 2). Families living in SSLP areas used more services designed to support child and family development than did those living in non-SSLP areas (table 2). In additional analyses, we tested whether SSLP effects on child outcomes were still significant if the parenting outcomes showing significant SSLP effects (home-learning environment and parenting risk) were added as predictors. These analyses showed that effects of SSLPs on parenting partly mediated the beneficial effect of SSLPS only on positive social behaviour (ie, 
SSLP to parenting to child), as shown by the substantial attenuation of the SSLP effect when negative parenting and home-learning environment were additional covariates.

For the SSLP versus non-SSLP comparisons, we had to exclude the most disadvantaged SSLP areas because none of the non-SSLP areas were comparably deprived. Could this exclusion have biased the SSLP effects we detected? To address this issue, children and families from SSLP areas included in the primary analyses were compared for all 14 outcomes with those from the SSLP areas excluded from the primary analyses. The differences were not significant (after adjustment for demographic variation), which suggests that exclusion of some SSLP areas did not bias the results and that the primary findings apply across all SSLP areas (ie, not just those included).

84 tests of two-way interactions indicated that SSLP effects did not vary substantially across demographic subgroups since only one interaction proved replicable across complete and imputed data, which is consistent with a chance result. Although SSLP effects on positive social behaviour were positive overall and for white children (ie, SSLP>non-SSLP), they were negative for black-Caribbean children (ie, non-SSLP $>$ SSLP), with no effect for other ethnic groups.

\section{Discussion}

After we took into consideration pre-existing background characteristics of children, families, and areas, and time of measurement, comparisons of children and families living in SSLP and non-SSLP areas showed five beneficial effects associated with living in SSLP areas and almost no evidence of adverse effects. Children growing up in these areas had better social development, with more positive social behaviour and greater independence than those in non-SSLP areas. In SSLP areas, risk of negative parenting was less than in non-SSLP areas and parents provided a more stimulating home-learning environment; these effects on parenting partly mediated the SSLP effect on positive social behaviour. These beneficial effects of SSLPs on children and their families might be related to the increased service use reported by parents in SSLP areas; therefore SSLPs are moving in the right direction. Even though the effect sizes were small to moderate, they were sufficiently large to be of policy significance in view of the fact that they applied on a population-wide basis, and are relevant to narrowing the gap in development between deprived children and the rest of the population.

The SSLP and non-SSLP areas that we compared were chosen by propensity matching of 85 area characteristics. Subsequent multilevel analyses also controlled for area characteristics. However, the local authorities where the SSLP and non-SSLP areas were situated might have differed, raising the possibility that differences between the areas were less likely to be a function of early-intervention programme than pre-existing localauthority differences. To explore this possibility, we examined local-authority differences between the SSLP and non-SSLP areas, even though SSLP areas typically included less than $10 \%$ of the population in the local authority. The local authorities of both areas did not differ in terms of geographic location (ie, nine government regions), but did so in terms of political control at the time data were gathered (ie, Labour, Conservative, Liberal Democrats, no overall control) since SSLP areas were more likely to be under no overall control (SSLP 27\%, non-SSLPs $11 \%, \chi^{2}$ test $\mathrm{p}=0 \cdot 01$ ). Local authorities covering SSLP and non-SSLP areas also differed in overall affluence (ie, Index of Multiple Deprivation, $\mathrm{p}=0 \cdot 03$ ); those with SSLP areas were more deprived. Of note, however, the non-SSLP areas were clearly within the range of deprivation of SSLP areas. Although the differences in local authorities are unlikely to explain our findings of beneficial effects associated with SSLPs, since we controlled for several area differences by propensity matching and covariates in analyses, they could partly account for why the non-SSLP areas were not designated to be SSLP areas at the time data were gathered despite being deprived.

The results of this second part of the evaluation differed substantially from those of the first part.' Some evidence of beneficial effects for most of the families living in SSLP areas was found in the previous assessment; however, some adverse effects were associated with living in SSLP areas for the most disadvantaged families (teenage parents, workless households, lone parents) compared with similar families in similarly disadvantaged areas not receiving SSLPs. This second part of the evaluation found no evidence of the adverse effects previously detected, despite explicitly testing for such subpopulation-specific effects in analyses. When beneficial effects associated with SSLPs were found, they could be generalised across population subgroups (eg, workless households, teenage mothers) in that there were almost no subgroup-specific SSLP effects. Children and families in SSLP areas that had been excluded from the primary comparisons functioned similarly to those in SSLP areas in the main comparisons. Such findings are consistent with the conclusion that the SSLP effects described can be generalised across the total population in all SSLPs areas in England.

In the early years of SSLPs, because of their local autonomy, there was little specification of how services were to be delivered, which was in sharp contrast to early interventions previously shown to be effective. ${ }^{2-5}$ The only other thorough evaluation of a community-based intervention for young children and their families with a similarly loose structure to that of the early SSLPS is the evaluation of a similar intervention in the USA, ${ }^{23}$ which showed no significant effect. The results of the initial evaluation of early SSLPs ${ }^{7}$ were mixed as already 
described. Since then SSLPs have developed into Sure Start Children's Centres with more clearly focused and specified services, and the results here indicate more beneficial effects for children and families. Evidence of the effectiveness of a particular focused approach to parenting has been provided for Sure Start programmes in Wales. ${ }^{24}$ Thus the evidence base suggests that a focused and specified approach to intervention in the child's early years is more likely to improve parenting and children's wellbeing.

A randomised controlled trial would have been the strongest evaluation strategy but government decisions precluded this possibility. The design we adopted was the most robust possible but study design alone should not decide the credibility of evidence about public-health interventions. ${ }^{25}$ We spent a great amount of effort on matching SSLP and non-SSLP areas, and only cases in areas where propensity matching showed comparable non-SSLP areas were used in the comparisons. Although all our analyses and estimates incorporated many covariates, we cannot rule out the possibility of unmeasured differences between the SSLP and non-SSLP groups. The proportion of missing data was low and the characteristics of cases with and without missing data were similar in both groups; moreover, to minimise missing data bias, we have only drawn attention to results that were significant for both complete and multiply-imputed data.

Methodological differences between the first and this second part of the SSLP-effect evaluation might have led to differences in results, notably the reliance in the second part on comparison data obtained by a different research team 2 years before. However, data gathered by the two teams were coordinated to ensure comparability for the analyses. We explicitly allowed for time of measurement effects in analyses testing for SSLP effects. Although we might not be as confident in the results from this quasi-experimental study as in results from a randomised controlled trial, the contrasting results might indicate the different experiences of children and families from SSLP areas participating in the two parts of the evaluation. SSLPs have evolved with time and become children's centres with more clearly focused services, more emphasis on children's development, and greater attention to the most vulnerable children and families-partly in response to the findings of the first part of the evaluation. ${ }^{1}$ With 7 years of acquired knowledge and experience, SSLPs have matured in their functioning, and staff-skills shortages have been reduced. Thus children and families in the present study might well have been exposed to more effective services than those encountered by children and families in the earlier phase of inquiry. Also the 3-year-old children in the earlier phase were seen when SSLPs had been set up for only 3 years, and economic analysis showed that the time taken for SSLPs to become fully functioning was 3 years. ${ }^{26}$ Hence SSLPs in the earlier phase would not have been providing bedded-down services for the entire lives of the children studied, whereas 3-year-old children and their families in the second part were exposed to more evolved and better developed programmes throughout the entire lives of the children.

\section{Contributors}

EM, JB, AL, and JBa participated in the conception and design of the study and obtaining funding. Members of the National Evaluation of Sure Start Research Team (Martin Frost, Gillian Harper, Department of Geography, Birkbeck University of London; Antero Malin,

Helena Romaniuk, Katrina Wilkins, Kristen McPherson, Jane Simons and Bina Ram, Institute for the Study of Children, Families and Social Issues, Birkbeck University of London) were involved in the acquisition of study data, doing statistical analyses, and supervising and training teams to gather data. EM and JB drafted the report with critical revision from AL and JBa. All authors have seen and approved the final version of the report for publication.

\section{Conflict of interest statement}

We declare that we have no conflict of interest.

\section{Acknowledgments}

The study was funded by the Department for Children, Schools and Families (DCSF) after competitive tender. The Medical Research Council Social and Public Health Sciences Unit is jointly funded by the Medical Research Council and the Chief Scientist Office of the Scottish Government Health Directorates (wbs U.1300.00.001).

\section{References}

1 Melhuish E, Hall D. The policy background to Sure Start. In: Belsky J, Barnes J, Melhuish E, eds. The national evaluation of Sure Start: does area-based early intervention work? Bristol: Policy Press 2007.

2 Ramey CT, Campbell FA, Burchinal M, et al. Persistent effects of early childhood education on high-risk children and their mothers. Appl Dev Sci 2000; 4: 2-14.

3 Olds D, Kitzman H, Cole R, et al. Effects of nurse home visiting on maternal life-course and child development: age-six follow-up of a randomized trial. Pediatrics 2004; 114: 1550-59.

4 Roberts C, Mazzucchelli T, Studman L, Sanders MR. A randomized control trial of behavioral family intervention for young children with developmental and behavioral problems. J Clin Child Adol Psychol 2006; 35: 180-93.

5 Love JM, Kisker EE, Ross C, et al. The effectiveness of Early Head Start for 3-year-old children and their parents: lessons for policy and programs. Dev Psychol 2005; 41: 885-901.

6 Belsky J, Barnes J, Melhuish E, eds. The national evaluation of Sure Start: does area-based early intervention work? Bristol: Policy Press, 2007.

7 Belsky J, Melhuish E, Barnes J, et al. Effects of Sure Start Loca Programmes on children and families: early findings from a quasi-experimental, cross-sectional study. BMJ 2006; 332: 1476-578.

8 Hansen K, ed. Millennium Cohort Study: first and second surveys. A guide to the datasets. London: IOE, 2006.

9 Rubin DB. Estimating causal effects from large datasets using propensity scores. Ann Int Med 1997; 127: 757-63.

10 The English indices of deprivation (revised). London: ODPM, 2004.

11 Elliot C, Smith P, McCulloch K. British Ability Scales, 2nd edn. Windsor: NFER-Nelson, 1996.

12 Goodman R. The strengths and difficulties questionnaire: a research note. J Child Psychol Psychiatry 1997; 38: 581-86.

13 Sammons P, Sylva K, Melhuish EC, et al. The effective provision of pre-school education project, technical paper 8b: measuring the impact on children's social behavioural development over the pre-school years. London: DfES, 2003.

14 Caldwell BM, Bradley RH. Home observation for measurement of the environment. Little Rock: University of Arkansas, 1984.

15 Pianta RC. The student-teacher relationship scale. Odessa: PAR, 2001. 
16 Straus MA, Hamby S, Finkelhor D, Moore D, Runyan D. Identification of child maltreatment with the parent-child conflict tactics scales: development and psychometric data for a national sample of American parents. Child Abuse Neglect 1998; 22: 249-70.

17 Matheny AP, Wachs T, Ludwig JL, Phillips K. Bringing order out of chaos: psychometric characteristics of the confusion, hubbub and order scale. J App Dev Psychol 1995; 16: 429-44.

18 Melhuish EC, Sylva K, Sammons P, et al. Effects of the home learning environment and preschool center experience upon literacy and numeracy development in early primary school. J Social Issues 2008; 64: 157-88.

19 Goldstein H. Multilevel statistical models, 2nd edn. London: Arnold, 1995.

20 Schafer JL. Analyses of incomplete multivariate data. London: Chapman and Hall, 1999.

21 Raghunathan TE, Solenberger PW, Van Hoewyk J. IVEware: imputation and variance estimation software. Ann Arbor: University of Michigan, 2007
22 Littell RC, Milliken GA, Stroup WW, Wolfinger RD,

Scabenberger O. SAS for mixed models, 2nd edn. Cary: SAS Institute, 2006

23 St Pierre RG, Layzer JI, Goodson BD, Bernstein LS. National impact evaluation of the comprehensive child development program. Washington, DC: US Department of Health and Human Services, 1997.

24 Hutchings J, Gardner F, Bywater T, et al. Parenting intervention in Sure Start services for children at risk of developing conduct disorder: pragmatic randomised controlled trial. BMJ 2007; 334: 678-82.

25 Rychetnik L, Frommer M, Hawe P, Shiell A. Criteria for evaluating evidence on public health interventions.

J Epidemiol Community Health 2002; 56: 119-127.

26 Meadows P. The costs and benefits of Sure Start Local Programmes. In: Belsky J, Barnes J, Melhuish E, eds. The national evaluation of Sure Start: does area-based early intervention work? Bristol: Policy Press, 2007. 